Acta Crystallographica Section E

Structure Reports

Online

ISSN 1600-5368

Editors: W. Clegg and D. G. Watson

\title{
4-lodo-1 $H$-pyrrole-2-carbaldehyde
}

Rohan A. Davis, Anthony R. Carroll, Ronald J. Quinn, Peter C. Healy and Alan R. White

Copyright $($ International Union of Crystallography

Author(s) of this paper may load this reprint on their own web site or institutional repository provided that this cover page is retained. Republication of this article or its storage in electronic databases other than as specified above is not permitted without prior permission in writing from the IUCr.

For further information see http://journals.iucr.org/services/authorrights.html 


\section{organic compounds}

Acta Crystallographica Section E

\section{Structure Reports}

Online

ISSN 1600-5368

\section{4-lodo-1H-pyrrole-2-carbaldehyde}

\section{Rohan A. Davis, Anthony R. Carroll, Ronald J. Quinn, Peter C. Healy* and Alan R. White}

Eskitis Institute for Cell and Molecular Therapies, Griffith University, Nathan, Brisbane 4111, Australia

Correspondence e-mail: p.healy@griffith.edu.au

Received 12 September 2007; accepted 12 September 2007

Key indicators: single-crystal X-ray study; $T=295 \mathrm{~K}$; mean $\sigma(\mathrm{C}-\mathrm{C})=0.006 \AA$ $R$ factor $=0.026 ; w R$ factor $=0.068 ;$ data-to-parameter ratio $=20.3$

The title compound, $\mathrm{C}_{5} \mathrm{H}_{4} \mathrm{INO}$, was synthesized during research aimed at producing suitable halogenated pyrrole building blocks for Suzuki-Miyaura coupling reactions. In the crystal structure, the molecules are planar and exhibit $\mathrm{N}-$ $\mathrm{H} \cdots \mathrm{O}$ bonding to form centrosymmetric dimers.

\section{Related literature}

For related literature, see: Davis et al. (2002); Mitsui et al. (2003); Miyaura \& Suzuki (1995); Monti \& Sleiter (1990); Smith et al. (1985); Sonnet (1972).

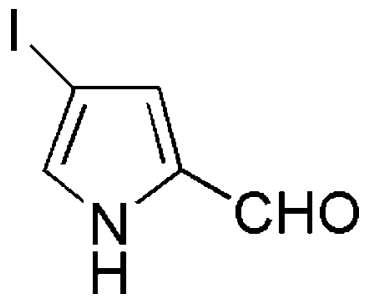

\section{Experimental}

Crystal data
$\mathrm{C}_{5} \mathrm{H}_{4} \mathrm{INO}$

$M_{r}=220.99$

Monoclinic, $P 2_{1} / c$

$a=10.245$ (3) ̊

$b=4.726$ (2) $\AA$

$c=13.531(4) \AA$

$\beta=92.73$ (2) ${ }^{\circ}$

\section{Data collection}

Rigaku AFC-7 $R$ diffractometer Absorption correction: $\psi$-scan

(North et al., 1968)

$T_{\min }=0.250, T_{\max }=0.533$

(expected range $=0.229-0.487$ )

1793 measured reflections

\section{Refinement}

$R\left[F^{2}>2 \sigma\left(F^{2}\right)\right]=0.026$

$w R\left(F^{2}\right)=0.068$

$S=1.11$

1505 reflections

1505 independent reflections 1281 reflections with $I>2 \sigma(I)$

$R_{\text {int }}=0.018$

3 standard reflections

every 150 reflections

intensity decay: $1.8 \%$

74 parameters

$\mathrm{H}$-atom parameters constrained

$\Delta \rho_{\max }=0.56 \mathrm{e} \mathrm{A}^{-3}$

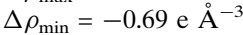

Table 1

Hydrogen-bond geometry $\left(\AA{ }^{\circ}\right)$.

\begin{tabular}{lllll}
\hline$D-\mathrm{H} \cdots A$ & $D-\mathrm{H}$ & $\mathrm{H} \cdots A$ & $D \cdots A$ & $D-\mathrm{H} \cdots A$ \\
\hline $\mathrm{N} 1-\mathrm{H} 1 \cdots \mathrm{O} 2^{\mathrm{i}}$ & 0.88 & 2.00 & $2.843(5)$ & 161 \\
$\mathrm{C} 5-\mathrm{H} 5 \cdots \mathrm{O} 2^{\text {ii }}$ & 0.95 & 2.50 & $3.400(5)$ & 158
\end{tabular}

Symmetry codes: (i) $-x+1,-y+2,-z$; (ii) $x,-y+\frac{3}{2}, z+\frac{1}{2}$.

Data collection: MSC/AFC7 Diffractometer Control Software (Molecular Structure Corporation, 1999); cell refinement: MSC/ AFC7 Diffractometer Control Software; data reduction: TEXSAN (Molecular Structure Corporation, 2001); program(s) used to solve structure: TEXSAN; program(s) used to refine structure: TEXSAN and SHELXL97 (Sheldrick, 1997); molecular graphics: ORTEP-3 (Farrugia, 1997); software used to prepare material for publication: TEXSAN and PLATON (Spek, 2003).

The authors acknowledge financial support of this work by Griffith University and the Eskitis Institute for Cell and Molecular Therapies.

Supplementary data and figures for this paper are available from the IUCr electronic archives (Reference: NG2326).

\section{References}

Davis, R. A., Carroll, A. R. \& Quinn, R. J. (2002). Aust. J. Chem. 55, 789-794. Farrugia, L. J. (1997). J. Appl. Cryst. 30, 565

Mitsui, T., Kimoto, M., Sato, A., Yokoyama, S. \& Hirao, I. (2003). Bioorg. Med. Chem. Lett. 13, 4515-4518.

Miyaura, N. \& Suzuki, A. (1995). Chem. Rev. 95, 2457-2483.

Molecular Structure Corporation (1999). MSC/AFC7 Diffractometer Control Software. Version 1.02 for Windows. MSC, The Woodlands, Texas, USA.

Molecular Structure Corporation (2001). TEXSAN. Version 1.06 for Windows.

MSC, The Woodlands, Texas, USA.

Monti, D. \& Sleiter, G. (1990). Gazz. Chim. Ital. 120, 771-774.

North, A. C. T., Phillips, D. C. \& Mathews, F. S. (1968). Acta Cryst. A24, 351359.

Sheldrick, G. M. (1997). SHELXL97. University of Göttingen, Germany. Smith, K. M., Bobe, F. W., Minnetian, O. M., Hope, H. \& Yanuck, M. D. (1985). J. Org. Chem. 50, 790-792.

Sonnet, P. E. (1972). J. Org. Chem. 37, 925-929.

Spek, A. L. (2003). J. Appl. Cryst. 36, 7-13. 


\section{supplementary materials}




\section{supplementary materials}

Acta Cryst. (2007). E63, o4076 [ doi:10.1107/S1600536807044534 ]

\section{4-Iodo-1H-pyrrole-2-carbaldehyde}

\section{R. A. Davis, A. R. Carroll, R. J. Quinn, P. C. Healy and A. R. White}

\section{Comment}

The title compound, (I), (Fig. 1) was synthesized during research aimed at producing suitable halogenated pyrrole building blocks for Suzuki-Miyaura coupling reactions (Miyaura \& Suzuki, 1995; Davis et al., 2002). Although compound (I) has been previously synthesized using a variety of methods (Mitsui et al., 2003; Monti \& Sleiter 1990; Sonnet, 1972) this is the first report of the X-ray crystal structure for 4-iodo- $1 \mathrm{H}$-pyrrole-2-carbaldehyde. As observed for related structures (Smith et al., 1985), the molecules are planar and exhibit $\mathrm{N}-\mathrm{H} \cdots \mathrm{O}$ bonding to form centrosymmetric dimers (Fig. 2).

\section{Experimental}

The commercial reagent pyrrole-2-carbaldehyde ( $475 \mathrm{mg}, 5 \mathrm{mmol}$ ) was added to an argon charged two-necked flask ( $50 \mathrm{ml}$ ) to which dry THF $(20 \mathrm{ml})$ was added and the mixture stirred for $10 \mathrm{~min}$ before being cooled to $195 \mathrm{~K}$. $\mathrm{N}$-iodosuccinimide (1.12 $\mathrm{g}, 5 \mathrm{mmol}$ ) was added portionwise over $15 \mathrm{~min}$ then the mixture was stirred at $195 \mathrm{~K}$ for $1 \mathrm{~h}$ before being transferred to a $258 \mathrm{~K}$ refrigerator for $16 \mathrm{~h}$. The solvent was removed under vacuum and the material was partitioned between $\mathrm{H}_{2} \mathrm{O}$ ( 50 $\mathrm{ml})$ and DCM $(2 \times 50 \mathrm{ml})$. The organic phase was dried $\left(\mathrm{MgSO}_{4}\right)$, then the DCM was evaporated under reduced pressure. The resulting residue was dissolved in DMSO $(10 \mathrm{ml})$ then chromatographed over a $\mathrm{C}_{18}$ flash column $(40 \mathrm{~mm} x 80 \mathrm{~mm})$ using $10 \%$ stepwise elutions from $20 \% \mathrm{MeOH} / 80 \% \mathrm{H}_{2} \mathrm{O}$ to $100 \% \mathrm{MeOH}$. The $70 \% \mathrm{MeOH} / 30 \% \mathrm{H}_{2} \mathrm{O}$ elution contained a 9:1 mixture of 4-iodo- $1 H$-pyrrole-2-carbaldehyde and 5-iodo- $1 H$-pyrrole-2-carbaldehyde $(165 \mathrm{mg})$, which proved to be inseparable by reversed-phase HPLC. Fractional crystallization using DCM/hexanes produced pure 4-iodo-1H-pyrrole-2carbaldehyde ( $72 \mathrm{mg}, 6.5 \%$ yield). Low yielding di-iodinated and tri-iodinated pyrrole derivatives were also detected during the purification work however no crystalline material was obtained for these compounds. NMR assignments for compound (I) were determined following analysis of the one-dimensional and two-dimensional NMR $(1 H, 13 \mathrm{C}, \mathrm{gCOSY}$, gHSQC, gHMBC) data.

4-iodo-1 $H$-pyrrole-2-carbaldehyde (I): clear needles, m.p. 390-391 K. ${ }^{1} \mathrm{H}$ NMR (DMSO-d 6 , $\left.600 \mathrm{MHz}\right) \delta 7.12$ (1H, s, $\mathrm{H}-3), 7.33$ (1H, s, H-5), 9.43 (1H, s, 2-CHO), 12.37 (1H, br s, 1-NH). ${ }^{13} \mathrm{C}$ NMR (DMSO-d $\left.6,150 \mathrm{MHz}\right) \delta 62.7$ (C-4), 126.0 (C-3), 131.3 (C-5), 134.4 (C-2), 178.7 (2-CHO). (-)-LRESIMS (rel. int.) m/z 220 (100\%) [M- $\left.\mathrm{H}_{2} \mathrm{C}_{5} \mathrm{H}_{3} \mathrm{INO}\right]^{-}$.

\section{Refinement}

The carbon-bound $\mathrm{H}$ atoms were constrained as riding atoms with $\mathrm{C}-\mathrm{H}=0.95-0.96 \AA$. The pyrrole proton was located in a difference Fourier synthesis and constrained with $\mathrm{N}-\mathrm{H}=0.88 \AA . U_{\text {iso }}(\mathrm{H})$ values were set at $1.2 U_{\text {eq }}$ of the parent atom. 


\section{supplementary materials}

\section{Figures}

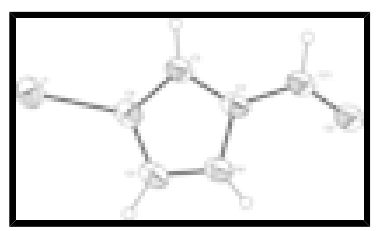

Fig. 1. View of (I) with the atom numbering scheme. Displacement ellipsoids for non-H atoms are drawn at the $30 \%$ probability level.

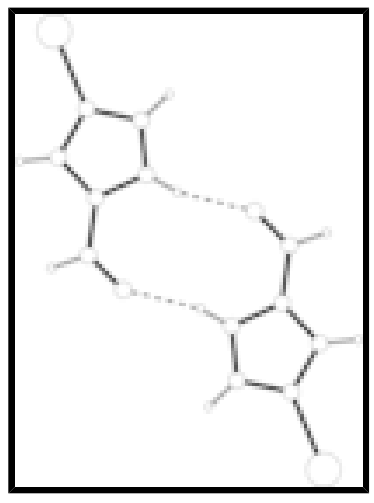

Fig. 2. View of the centrosymmetric dimers of (I).

\section{4-iodo-1H-pyrrole-2-carbaldehyde}

Crystal data

\section{$\mathrm{C}_{5} \mathrm{H}_{4} \mathrm{INO}$}

$M_{r}=220.99$

Monoclinic, $P 2_{1} / c$

Hall symbol: -P 2ybc

$a=10.245$ (3) $\AA$

$b=4.726(2) \AA$

$c=13.531(4) \AA$

$\beta=92.73(2)^{\circ}$

$V=654.4(4) \AA^{3}$

$Z=4$

\section{Data collection}

Rigaku AFC-7R diffractometer

Radiation source: Rigaku rotating anode

Monochromator: graphite

$T=295 \mathrm{~K}$

$\omega / 2 \theta$ scans

Absorption correction: $\psi$ scan

(North et al., 1968)

$T_{\min }=0.250, T_{\max }=0.533$

1793 measured reflections

1505 independent reflections
$F_{000}=408$

$D_{\mathrm{x}}=2.243 \mathrm{Mg} \mathrm{m}^{-3}$

Mo $K \alpha$ radiation

$\lambda=0.71069 \AA$

Cell parameters from 25 reflections

$\theta=12.5-16.3^{\circ}$

$\mu=4.79 \mathrm{~mm}^{-1}$

$T=295$ (2) K

Plate, colourless

$0.40 \times 0.40 \times 0.15 \mathrm{~mm}$

$R_{\text {int }}=0.018$

$\theta_{\max }=27.5^{\circ}$

$\theta_{\min }=3.0^{\circ}$

$h=-13 \rightarrow 13$

$k=-2 \rightarrow 6$

$l=-7 \rightarrow 17$

3 standard reflections every 150 reflections intensity decay: $1.8 \%$ 


\section{supplementary materials}

1281 reflections with $I>2 \sigma(I)$

\section{Refinement}

Refinement on $F^{2}$

Least-squares matrix: full

$R\left[F^{2}>2 \sigma\left(F^{2}\right)\right]=0.026$

$w R\left(F^{2}\right)=0.068$

$S=1.11$

1505 reflections

Hydrogen site location: inferred from neighbouring

sites

$\mathrm{H}$-atom parameters constrained

$$
w=1 /\left[\sigma^{2}\left(F_{\mathrm{o}}{ }^{2}\right)+(0.0254 P)^{2}+0.8517 P\right]
$$

where $P=\left(F_{\mathrm{o}}{ }^{2}+2 F_{\mathrm{c}}{ }^{2}\right) / 3$

$(\Delta / \sigma)_{\max }<0.001$

$\Delta \rho_{\max }=0.56 \mathrm{e} \AA^{-3}$

$\Delta \rho_{\min }=-0.69$ e $\AA^{-3}$

74 parameters

Extinction correction: SHELXL97 (Sheldrick, 1997),

$\mathrm{Fc}^{*}=\mathrm{kFc}\left[1+0.001 \mathrm{Fc}^{2} \lambda^{3} / \sin (2 \theta)\right]^{-1 / 4}$

Primary atom site location: structure-invariant direct methods

Extinction coefficient: 0.0057 (6)

Secondary atom site location: difference Fourier map

\section{Special details}

Experimental. The scan width was $(1.79+0.30 \tan \theta)^{\circ}$ with an $\omega$ scan speed of $16^{\circ}$ per minute (up to 5 scans to achieve $\mathrm{I} / \sigma(\mathrm{I})>10$ ). Stationary background counts were recorded at each end of the scan, and the scan time:background time ratio was 2:1.

Geometry. Bond distances, angles etc. have been calculated using the rounded fractional coordinates. All su's are estimated from the variances of the (full) variance-covariance matrix. The cell e.s.d.'s are taken into account in the estimation of distances, angles and torsion angles

Refinement. Refinement on $\mathrm{F}^{2}$ for ALL reflections except those flagged by the user for potential systematic errors. Weighted $R$-factors $w R$ and all goodness of fit values $\mathrm{S}$ are based on $\mathrm{F}^{2}$, conventional $R$-factors $R$ are based on $\mathrm{F}$, with $\mathrm{F}$ set to zero for negative $\mathrm{F}^{2}$. The observed criterion of $\mathrm{F}^{2}>2 \operatorname{sigma}\left(\mathrm{F}^{2}\right)$ is used only for calculating $-R$-factor-obs $e t c$. and is not relevant to the choice of reflections for refinement. $R$-factors based on $\mathrm{F}^{2}$ are statistically about twice as large as those based on $\mathrm{F}$, and $R$-factors based on ALL data will be even larger.

Fractional atomic coordinates and isotropic or equivalent isotropic displacement parameters $\left(\AA^{2}\right)$

$\begin{array}{lllll} & x & y & z & U_{\text {iso }} * / U_{\text {eq }} \\ \mathrm{I} 1 & 0.10101(3) & 0.11438(6) & 0.17038(2) & 0.0593(1) \\ \mathrm{O} 2 & 0.4065(3) & 0.9850(9) & -0.1241(2) & 0.0696(10) \\ \mathrm{N} 1 & 0.6731(8) & 0.0582(2) & 0.0536(10) \\ \mathrm{C} 2 & 0.3783(3) & -0.0239(3) & 0.0521(13) \\ \mathrm{C} 3 & 0.2945(4) & 0.4801(10) & -0.0045(3) & 0.0531(11) \\ \mathrm{C} 4 & 0.1952(4) & 0.3788(8) & 0.0908(3) & 0.0490(11) \\ \mathrm{C} 5 & 0.2196(4) & 0.5006(10) & 0.1282(3) & 0.0547(13) \\ \mathrm{C} 21 & 0.3333(4) & 0.8263(11) & -0.1112(3) & 0.0601(14) \\ \mathrm{H} 1 & 0.3154(4) & 0.77370 & 0.06420 & 0.0630^{*} \\ \mathrm{H} 2 & 0.45070 & 0.80760 & -0.16460 & 0.0710^{*} \\ \mathrm{H} 3 & 0.25140 & 0.46580 & -0.04790 & 0.0630 * \\ \mathrm{H} 5 & 0.12300 & 0.19280 & 0.0640 *\end{array}$




\section{supplementary materials}

Atomic displacement parameters $\left(\hat{A}^{2}\right)$

$\begin{array}{lllllll} & U^{11} & U^{22} & U^{33} & U^{12} & U^{13} & U^{23} \\ \mathrm{I} 1 & 0.0656(2) & 0.0567(2) & 0.0560(2) & 0.0093(1) & 0.0078(1) & 0.0053(1) \\ \mathrm{O} 2 & 0.0663(18) & 0.096(2) & 0.0456(15) & -0.0168(18) & -0.0061(13) & 0.0071(17) \\ \mathrm{N} 1 & 0.0476(16) & 0.071(2) & 0.0417(16) & 0.0030(16) & -0.0029(13) & -0.0036(16) \\ \mathrm{C} 2 & 0.053(2) & 0.065(3) & 0.0376(17) & 0.0046(19) & -0.0053(15) & -0.0039(17) \\ \mathrm{C} 3 & 0.053(2) & 0.062(2) & 0.0435(19) & -0.0021(19) & -0.0067(16) & -0.0025(19) \\ \mathrm{C} 4 & 0.0533(19) & 0.052(2) & 0.0418(18) & 0.0109(17) & 0.0035(15) & -0.0023(17) \\ \mathrm{C} 5 & 0.056(2) & 0.069(3) & 0.0387(18) & 0.013(2) & -0.0017(16) & -0.0012(19) \\ \mathrm{C} 21 & 0.058(2) & 0.081(3) & 0.0404(19) & -0.008(2) & -0.0070(16) & 0.002(2)\end{array}$

Geometric parameters $\left(A,{ }^{\circ}\right)$

\begin{tabular}{|c|c|c|c|}
\hline $\mathrm{I} 1-\mathrm{C} 4$ & $2.079(4)$ & $\mathrm{C} 2-\mathrm{C} 21$ & $1.436(6)$ \\
\hline $\mathrm{O} 2-\mathrm{C} 21$ & $1.216(6)$ & $\mathrm{C} 3-\mathrm{C} 4$ & $1.387(6)$ \\
\hline $\mathrm{N} 1-\mathrm{C} 2$ & $1.372(5)$ & $\mathrm{C} 4-\mathrm{C} 5$ & $1.374(6)$ \\
\hline $\mathrm{N} 1-\mathrm{C} 5$ & $1.348(5)$ & $\mathrm{C} 3-\mathrm{H} 3$ & 0.9500 \\
\hline $\mathrm{N} 1-\mathrm{H} 1$ & 0.8800 & $\mathrm{C} 5-\mathrm{H} 5$ & 0.9500 \\
\hline $\mathrm{C} 2-\mathrm{C} 3$ & $1.369(6)$ & $\mathrm{C} 21-\mathrm{H} 2$ & 0.9600 \\
\hline $\mathrm{I} 1 \cdots \mathrm{C} 4^{\mathrm{i}}$ & $3.853(4)$ & $\mathrm{C} 4 \cdots \mathrm{I} 1^{\mathrm{vii}}$ & $3.853(4)$ \\
\hline $\mathrm{I} 1 \cdots \mathrm{C} 5^{\mathrm{i}}$ & $3.812(5)$ & $\mathrm{C} 5 \cdots \mathrm{I} 1^{\mathrm{vii}}$ & $3.812(5)$ \\
\hline $\mathrm{I} 1 \cdots \mathrm{I} 1^{\mathrm{ii}}$ & $3.8646(17)$ & $\mathrm{C} 5 \cdots \mathrm{O} 2^{\text {viii }}$ & $3.400(5)$ \\
\hline $\mathrm{I} 1 \cdots \mathrm{I}^{\mathrm{iii}}$ & $3.8646(17)$ & $\mathrm{C} 21 \cdots \mathrm{H} 1^{\mathrm{vi}}$ & 3.0900 \\
\hline $\mathrm{I} 1 \cdots \mathrm{H} 2^{\mathrm{iv}}$ & 3.3200 & $\mathrm{C} 21 \cdots \mathrm{H} 5^{\mathrm{v}}$ & 2.9100 \\
\hline $\mathrm{O} 2 \cdots \mathrm{N} 1$ & $2.900(5)$ & $\mathrm{H} 1 \cdots \mathrm{O} 2$ & 2.7500 \\
\hline $\mathrm{O} 2 \cdots \mathrm{C} 5^{\mathrm{v}}$ & $3.400(5)$ & $\mathrm{H} 1 \cdots \mathrm{O} 2^{\mathrm{vi}}$ & 2.0000 \\
\hline $\mathrm{O} 2 \cdots \mathrm{N} 1^{\mathrm{vi}}$ & $2.843(5)$ & $\mathrm{H} 1 \cdots \mathrm{C} 21^{\mathrm{vi}}$ & 3.0900 \\
\hline $\mathrm{O} 2 \cdots \mathrm{H} 1^{\mathrm{vi}}$ & 2.0000 & $\mathrm{H} 2 \cdots \mathrm{I} 1^{\mathrm{ix}}$ & 3.3200 \\
\hline $\mathrm{O} 2 \cdots \mathrm{H} 1$ & 2.7500 & $\mathrm{H} 2 \cdots \mathrm{H} 5^{\mathrm{v}}$ & 2.5700 \\
\hline $\mathrm{O} 2 \cdots \mathrm{H}^{\mathrm{v}}$ & 2.5000 & $\mathrm{H} 5 \cdots \mathrm{O} 2^{\text {viii }}$ & 2.5000 \\
\hline $\mathrm{N} 1 \cdots \mathrm{O} 2$ & $2.900(5)$ & $\mathrm{H} 5 \cdots \mathrm{C} 21^{\mathrm{viii}}$ & 2.9100 \\
\hline $\mathrm{N} 1 \cdots \mathrm{O} 2^{\mathrm{vi}}$ & $2.843(5)$ & $\mathrm{H} 5 \cdots \mathrm{H} 2^{\text {viii }}$ & 2.5700 \\
\hline $\mathrm{C} 2-\mathrm{N} 1-\mathrm{C} 5$ & $109.0(3)$ & $\mathrm{C} 3-\mathrm{C} 4-\mathrm{C} 5$ & $108.1(4)$ \\
\hline $\mathrm{C} 5-\mathrm{N} 1-\mathrm{H} 1$ & 125.00 & $\mathrm{~N} 1-\mathrm{C} 5-\mathrm{C} 4$ & $107.9(3)$ \\
\hline $\mathrm{C} 2-\mathrm{N} 1-\mathrm{H} 1$ & 126.00 & $\mathrm{O} 2-\mathrm{C} 21-\mathrm{C} 2$ & $126.5(4)$ \\
\hline $\mathrm{N} 1-\mathrm{C} 2-\mathrm{C} 21$ & $122.3(4)$ & $\mathrm{C} 2-\mathrm{C} 3-\mathrm{H} 3$ & 127.00 \\
\hline $\mathrm{N} 1-\mathrm{C} 2-\mathrm{C} 3$ & $108.0(4)$ & $\mathrm{C} 4-\mathrm{C} 3-\mathrm{H} 3$ & 126.00 \\
\hline $\mathrm{C} 3-\mathrm{C} 2-\mathrm{C} 21$ & $129.7(4)$ & $\mathrm{N} 1-\mathrm{C} 5-\mathrm{H} 5$ & 128.00 \\
\hline $\mathrm{C} 2-\mathrm{C} 3-\mathrm{C} 4$ & $107.1(4)$ & $\mathrm{C} 4-\mathrm{C} 5-\mathrm{H} 5$ & 124.00 \\
\hline $\mathrm{I} 1-\mathrm{C} 4-\mathrm{C} 5$ & $124.6(3)$ & $\mathrm{O} 2-\mathrm{C} 21-\mathrm{H} 2$ & 117.00 \\
\hline $\mathrm{I} 1-\mathrm{C} 4-\mathrm{C} 3$ & $127.2(3)$ & $\mathrm{C} 2-\mathrm{C} 21-\mathrm{H} 2$ & 117.00 \\
\hline $\mathrm{C} 5-\mathrm{N} 1-\mathrm{C} 2-\mathrm{C} 3$ & $0.0(5)$ & $\mathrm{C} 3-\mathrm{C} 2-\mathrm{C} 21-\mathrm{O} 2$ & $-179.7(5)$ \\
\hline $\mathrm{C} 5-\mathrm{N} 1-\mathrm{C} 2-\mathrm{C} 21$ & $179.5(4)$ & $\mathrm{C} 2-\mathrm{C} 3-\mathrm{C} 4-\mathrm{I} 1$ & $175.5(3)$ \\
\hline $\mathrm{C} 2-\mathrm{N} 1-\mathrm{C} 5-\mathrm{C} 4$ & $-0.1(5)$ & $\mathrm{C} 2-\mathrm{C} 3-\mathrm{C} 4-\mathrm{C} 5$ & $0.0(5)$ \\
\hline
\end{tabular}




\section{supplementary materials}

$\begin{array}{lccc}\mathrm{N} 1-\mathrm{C} 2-\mathrm{C} 3-\mathrm{C} 4 & 0.0(5) & \mathrm{I} 1-\mathrm{C} 4-\mathrm{C} 5-\mathrm{N} 1 & -175.6(3) \\ \mathrm{C} 21-\mathrm{C} 2-\mathrm{C} 3-\mathrm{C} 4 & -179.4(4) & \mathrm{C} 3-\mathrm{C} 4-\mathrm{C} 5-\mathrm{N} 1 & 0.1(5) \\ \mathrm{N} 1-\mathrm{C} 2-\mathrm{C} 21-\mathrm{O} 2 & 1.1(7) & \\ \text { Symmetry codes: (i) } x, y-1, z ;(\mathrm{ii})-x, y-1 / 2,-z+1 / 2 ;(\mathrm{iii})-x, y+1 / 2,-z+1 / 2 ;(\mathrm{iv}) x,-y+1 / 2, z+1 / 2 ;(\mathrm{v}) x,-y+3 / 2, z-1 / 2 ;(\mathrm{vi})-x+1, \\ -y+2,-z ;(\text { vii } x, y+1, z ;(\text { viii) } x,-y+3 / 2, z+1 / 2 ;(\mathrm{ix}) x,-y+1 / 2, z-1 / 2 .\end{array}$

Hydrogen-bond geometry $\left(A,{ }^{\circ}\right)$

$\begin{array}{lllll}D-\mathrm{H} \cdots A & D-\mathrm{H} & \mathrm{H} \cdots A & D \cdots A & D-\mathrm{H} \cdots A \\ \mathrm{~N} 1-\mathrm{H} 1 \cdots \mathrm{O} 2^{\mathrm{vi}} & 0.88 & 2.00 & 2.843(5) & 161 \\ \mathrm{C} 5-\mathrm{H} 5 \cdots \mathrm{O} 2^{\text {viii }} & 0.95 & 2.50 & 3.400(5) & 158\end{array}$

Symmetry codes: (vi) $-x+1,-y+2,-z$; (viii) $x,-y+3 / 2, z+1 / 2$. 
Fig. 1

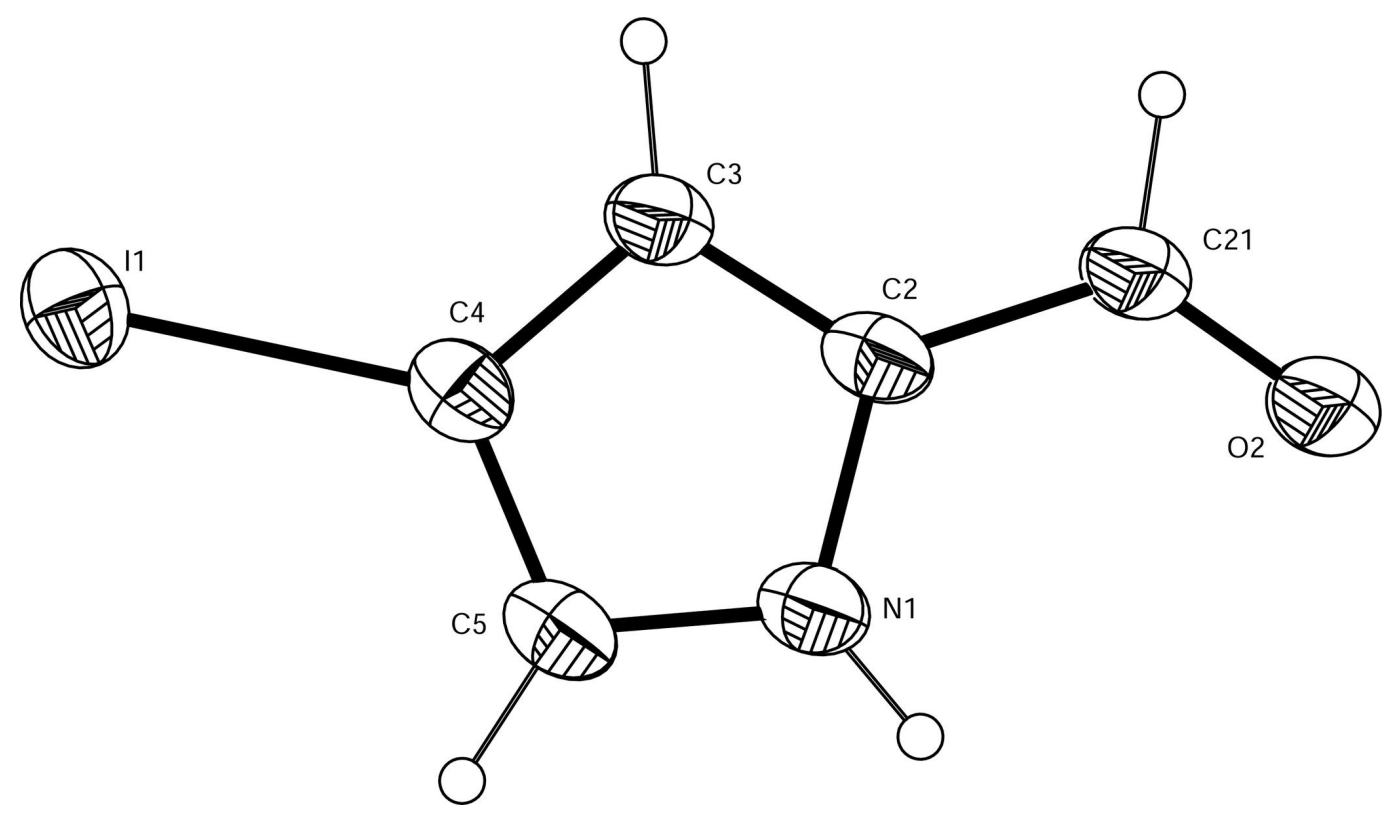


Fig. 2

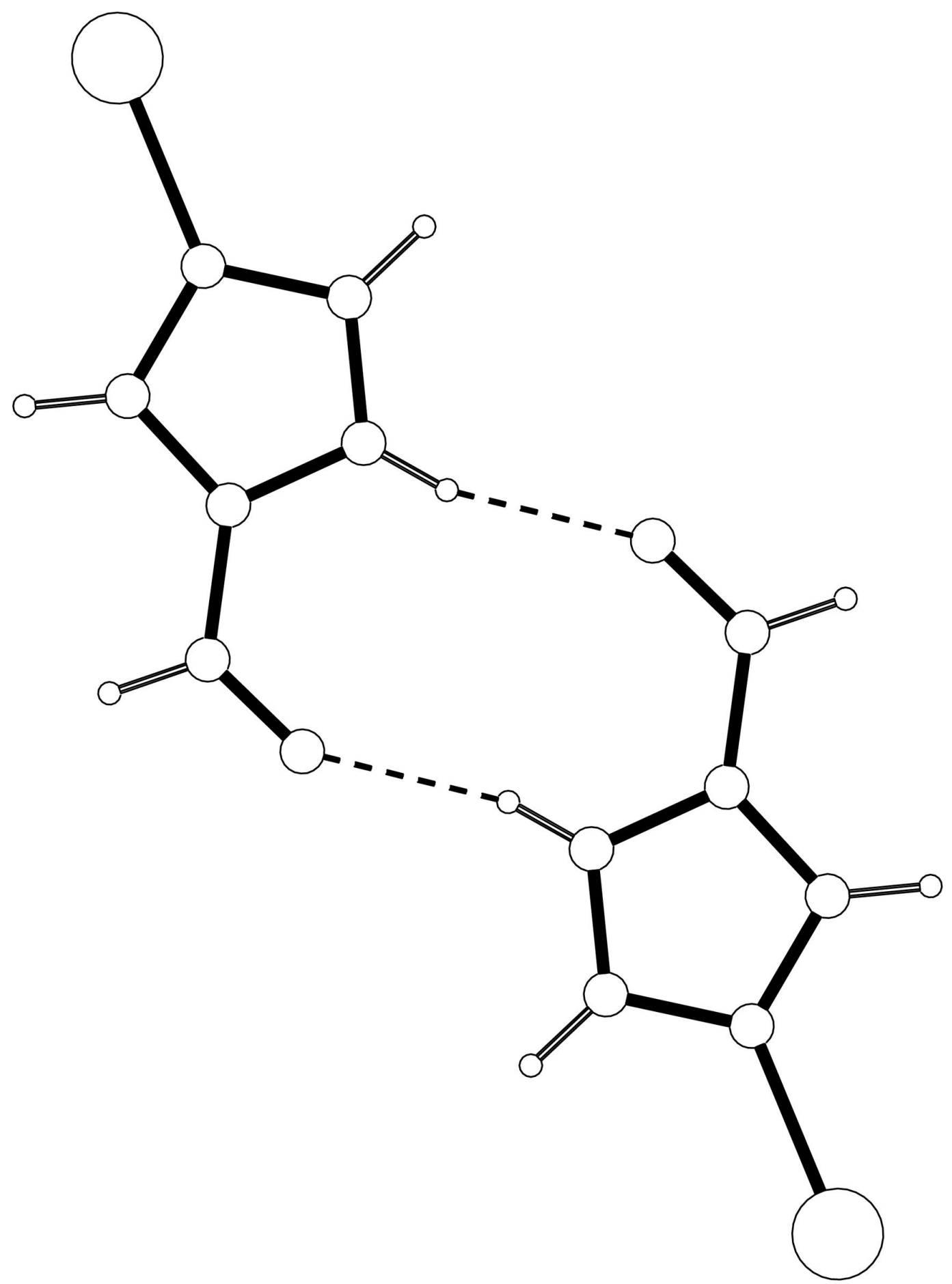

